\section{SAT0110 SARCOPENIA IN PATIENTS WITH RHEUMATOID ARTHRITIS ON THE TREATMENT WITH BIOLOGIC DISEASE MODIFYING ANTI-RHEUMATIC DRUGS}

E. Hasegawa ${ }^{1,2}$, S. Ito ${ }^{1}$, Y. Kurosawa ${ }^{1,2}$, S. Taniguchi ${ }^{1}$, D. Kobayashi ${ }^{1,2}$, A. Abe ${ }^{1}$, H. Otani ${ }^{1}$, K. Nakazono ${ }^{1}$, A. Murasawa ${ }^{1}$, I. Narita ${ }^{2}$, H. Ishikawa ${ }^{1} .{ }^{1}$ Niigata Rheumatic Center, Department of Rheumatology, Niigata, Japan: ${ }^{2}$ Niigata University Graduate School of Medical and Dental Sciences, Division of Clinical Nephrology and Rheumatology, Niigata, Japan

Background: Sarcopenia is characterized by loss of muscle mass and strength, which lead to lower physical ability, less quality of life (QoL), frailty and mortality. Rheumatoid arthritis (RA) is considered to be one of the causes of sarcopenia. Objectives: To clarify the effectiveness of biologic disease modifying anti-rheumatic drugs (bDMARDs) on sarcopenia, including physical ability, body composition and nutritional status.

Methods: This is a prospective cohort study including consecutive 48 patients (male 11, female 37, age 64.2 \pm 15.1 ) with RA who started bDMARDs in Niigata Rheumatic Center. Diagnosis of sarcopenia was according to the diagnostic algorithm of European Working Group on Sarcopenia in Older People (EWGSOP). We monitored disease activity of RA, physical ability, body composition, nutritional status and QoL at baseline, 6 months and at 12 months. Disease activity was measured by disease activity score-28 joint count based on erythrocyte sedimentation rate (DAS28-ESR), clinical disease activity index (CDAl). Physical activity was measured by Health Assessment Questionnaire (HAQ), 10m walking test (10MWT). Nutritional status was measured by controlling nutrition status (CONUT) score, and prognostic nutritional index (PNI). Overall QoL was measured by EuroQol 5 dimentions (EQ5D).

Results: Among 48 patients who started bDMARDs, 21 patients were classified as having sarcopenia. The bDMARDs used were adalimumab in 10 cases, certolizumab pegol in 9 cases, abatacept in 9 cases, golimumab in 7 cases, tocilizumab in 5 cases, infliximab in 5 cases and etanercept in 3 cases. DAS28-ESR $(4.7 \pm 1.4$ vs. $2.7 \pm 1.0$, $p<0.001)$ and CDAl $(18.4 \pm 9.4$ vs. $7.4 \pm 5.5, p<0.001)$ were significantly decreased by 12 months of bDMARDs therapy. Physical activity was significantly ameliorated after 12 months of bDMARDs; $\mathrm{HAQ}(1.1 \pm 0.9$ vs. $0.6 \pm 0.8, \mathrm{p}<0.001), 10 \mathrm{MWT}(1.5 \pm 0.7$ $\mathrm{m} / \mathrm{s}$ vs. $1.7 \pm 0.6, p=0.002)$. $E Q-5 D$ was also ameliorated $(0.63 \pm 0.15$ vs. $0.74 \pm 0.19$, $\mathrm{p}=0.0002$ ). As for body composition analysis, there were significant increase in body weight $(54.6 \pm 12.4 \mathrm{~kg}$ vs. $55.8 \pm 13.6, \mathrm{p}=0.006)$, but there was no significant increase in skeletal muscle mass index $(5.9 \pm 1.1 \mathrm{~kg} / \mathrm{m} 2$ vs. $5.9 \pm 1.1, \mathrm{p}=0.229)$. Among 21 patients who were classified as sarcopenia when starting bDMARDs, the number of patients having sarcopenia significantly decreased after 12 months of bDMARDs ( $100 \%$ vs. $52.3 \%, \mathrm{p}=0.0005)$ and skeletal muscle index of these patients were significantly increased $(5.1 \pm 0.5 \mathrm{~kg} / \mathrm{m} 2$ vs. $5.3 \pm 0.7, \mathrm{p}=0.046)$.

Conclusion: Twelve months of bDMARDs therapy significantly ameliorated disease activity, nutritional status and physical activity. In RA patients with sarcopenia, bDMARDs significantly increased skeletal muscle and may be effective for treatment of sacrcopenia.

Disclosure of Interests: : Eriko Hasegawa: None declared, Satoshi Ito Speakers bureau: Abbvie,Eisai, Yoichi Kurosawa: None declared, Shinji Taniguchi: None declared, Daisuke Kobayashi: None declared, Asami Abe: None declared, Hiroshi Otani: None declared, Kiyoshi Nakazono: None declared, Akira Murasawa: None declared, Ichiei Narita: None declared, Hajime Ishikawa: None declared DOI: 10.1136/annrheumdis-2020-eular.1036

\section{\begin{tabular}{l|l}
\hline SAT0111 & THE RELATIONSHIP BETWEEN THE ADMINISTRATION
\end{tabular} OF IL-6 INHIBITORS AND INSULIN RESISTANCE IN PATIENTS WITH ACTIVE RHEUMATOID ARTHRITIS}

A. Jitaru ${ }^{1}$, C. Pomirleanu' ${ }^{2}$, M. M. Leon-Constantin ${ }^{3}$, F. Mitu ${ }^{3}$, C. Ancuta ${ }^{2}$. ${ }^{1}$ Grigore T. Popa University of Medicine and Pharmacy, Faculty of Medicine, lasi, Romania; ${ }^{2}$ Clinical Rehabilitation Hospital, 2nd Rheumatology Clinic, lasi, Romania; ${ }^{3}$ Clinical Rehabilitation Hospital, Cardiovascular Rehabilitation Clinic, lasi, Romania

Background: Rheumatoid arthritis (RA) is associated with an increased cardiovascular (CV) risk, due not only to the traditional risk factors (hypertension, insulin resistance/diabetes, obesity, smoking), but to the inflammatory status as well. The blockade of interleukin-6 (IL-6) can regulate the glucose metabolism, reducing the glucose level and insulin resistance (IR). This beneficial effect is seen more in patients with normal values of body mass index (BMI), compared to the obese population.

Objectives: Given the mentioned existing data, we aim to demonstrate the positive effect of IL-6 inhibitors in active RA patients with normal or increased BMI. Methods: We recruited 56 consecutive patients with definite and active RA, non-responders/partial responders to conventional synthetic Drug Modifying Anti-Rheumatic Drugs (csDMARDs)/biological therapy. For a period of 52 weeks, patients received subcutaneous Tocilizumab (TCZ) in a dose of 162mg once a week, according to European League Anti Rheumatism (EULAR) recommendation and National Protocol. We assessed demographics, RA-related parameters (clinical, inflammatory and immune) and metabolic markers, as well as the peripheral response to insulin, quantified by Homeostasis Model Assessment for insulin resistance (HOMA-IR) and the Quantitative Insulin Sensitivity Check Index (QUICKI). We did not include in the study the patients known with diabetes mellitus (DM) and those undergoing glucocorticoids.

Results: After 52 weeks of treatment, most of the patients showed a statistically significant reduction of HOMA-IR $(3.61 \pm 1.21$ at the onset vs. $2.45 \pm 1.46$ at the end of the study, $p<0.001)$, while QUICKI registered a slight increase $(0.32 \pm 0.01$ at the onset vs. $0.33 \pm 0.01$ at the end of the study, $p<0.001$ ). Also, the decrease in insulin and glucose levels were more obvious in patients with normal BMI, strictly related to disease activity.

Conclusion: Long-term administration of TCZ in active RA is associated with a significant reduction of disease activity and IR, especially in normal weight patients. This confirms that obesity, as a $\mathrm{CV}$ risk factor, represents one of the main causes of IR

\section{References:}

[1] Castañeda S, Remuzgo-Martínez S, López-Mejías R et al. Rapid beneficial effect of the IL-6 receptor blockade on insulin resistance and insulin sensitivity in non-diabetic patients with rheumatoid arthritis. Clin Exp Rheumatol. 2019; 37(3):465-473.

[2] Lehrskov LL, Christensen RH. The role of interleukin-6 in glucose homeostasis and lipid metabolism. Semin Immunopathol. 2019; 41(4):491-499.

[3] Ursini F, Russo E, Ruscitti P, Giacomelli R, De Sarro G. The effect of non-TNFtargeted biologics and small molecules on insulin resistance in inflammatory arthritis. Autoimmun Rev. 2018 Apr;17(4):399-404.

Disclosure of Interests: Alexandra Jitaru: None declared, Cristina Pomirleanu: None declared, Maria-Magdalena Leon-Constantin: None declared, Florin Mitu: None declared, CODRINA ANCUTA Consultant of: AbbVie, Pfizer, Roche, Novartis, UCB, Ewopharma, Merck Sharpe and Dohme, and Eli Lilly, Speakers bureau: AbbVie, Pfizer, Roche, Novartis, UCB, Ewopharma, Merck Sharpe and Dohme, and Eli Lilly

DOI: 10.1136/annrheumdis-2020-eular.6509

\section{\begin{tabular}{l|l} 
SAT0112 & EFFECT OF RITUXIMAB ON IMMUNOGLOBULIN
\end{tabular} LEVELS AND RISK OF ASSOCIATED INFECTION}

S. Khalid ${ }^{1}$, R. Smith ${ }^{2}$, L. Yalakki ${ }^{1}{ }^{1}$ Basingstoke and North Hampshire Hospital, Rheumatology, Basingstoke, United Kingdom; ${ }^{2}$ Salisbury District Hospital, Salisbury, United Kingdom

Background: Rituximab (RTX) is an anti-CD20 monoclonal antibody that suppresses B-lymphocytes and may induce hypogammaglobulinemia. Studies have shown that sustained low levels of immunoglobulins $(\mathrm{lg})$ are associated with significantly increased risks of infections.

Objectives: To determine the relationship between the serum lg levels and risk of infection during (RTX) therapy for rheumatic diseases. We also aimed to identify the most common type of infections and pathogens associated with them.

Methods: A multi-centre retrospective observational study of patients with autoimmune diseases treated with RTX between 2009-2019. Serum Ig levels (IgM, IgG and $\lg A$ ) were measured at baseline and 6-12 months after each cycle and note was made of any hypogammaglobulianemia. Infections, evidenced by a positive microbiology sample, radiograph or requirement of antibiotics and/or hospitalization, were recorded.

Results: 146 patients were included with a mean age of 61.5 years (standard deviation 13.8). 105 patients $(71.9 \%)$ ) had Rheumatoid Arthritis. 51/146 had had 1or more episodes of infections whilst on Rituximab which required treatment. Of these, $26(50.9 \%)$ had recurrent infections. 33/51 had low immunoglobulins of at least 1 type. After receiving RTX treatment, 14 had low IgG, 29 had low IgM and only 4 had low IgA. 6 patients with low IgG, but 13 patients with low IgM suffered with recurrent infections.

There was a statistically significant higher proportion of patients with infection who had low Ig levels compared to those with normal levels, with a $p<0.01$ on chi square testing.

Lower respiratory tract infections were the commonest infection seen with 28 cases, with most cases (15/28) caused by Haemophilus Influenza. 18 cases of urinary tract infections were seen, with 12/18 being caused by E. Coli. 3 cases of shingles were also seen.

Conclusion: Our study shows that an increased risk of infection was associated with hypogammaglobulinemia after rituximab therapy and highlights the importance of monitoring these patients. In our data set, more patients with recurrent infections had low IgM, supporting a need for better understanding of low IgM and its relation to infection. Respiratory tract infections were the most common infection with Haemophilus Influenza being the commonest pathogen. Recent studies have shown an increase in haemophilus influenza infections. Reasons for the increase may include a waning immunity to Haemophilus, changes in the organism and greater numbers of high-risk people- such as in our study. This supports a rationale 
for extended indication for immunisation against Haemophilus in vulnerable groups of adults and has implications for targeted adult Haemophilus influenzae vaccine development.

\begin{tabular}{|l|l|l|l|}
\hline & Infection & Uninfected & Marginal Row Totals \\
\hline Normal IG & $18(26.2)[2.57]$ & $57(48.8)[1.38]$ & 75 \\
\hline Low IG & $33(24.8)[2.71]$ & $38(46.2)[1.45]$ & 71 \\
\hline Marginal Column Totals & 51 & 95 & 146 (Grand Total) \\
\hline
\end{tabular}

The chi-square statistic is 8.1083 . The $p$-value is .004406 . Significant at $p<.05$.

The chi-square statistic with Yates correction is 7.1494 . The $p$-value is .007499 . Significant at $p<.05$.

\section{References:}

[1] Kridin K, Ahmed AR. Post-rituximab immunoglobulin M (IgM) hypogammaglobulinemia. Autoimmunity Reviews. 2020 Jan 6:102466.

[2] Barmettler S, Ong MS, Farmer JR, Choi H, Walter J. Association of Immunoglobulin Levels, Infectious Risk, and Mortality With Rituximab and Hypogammaglobulinemia. JAMA Netw Open. 2018;1(7):e184169. Published 2018 Nov 2.

[3] Casulo C, Maragulia J, Zelenetz AD. Incidence of hypogammaglobulinemia in patients receiving rituximab and the use of intravenous immunoglobulin for recurrent infections. Clin Lymphoma Myeloma Leuk. 2013;13(2): 106-111.

[4] Nix EB, Hawdon N, Gravelle S, et al. Risk of invasive Haemophilus influenzae type $\mathrm{b}(\mathrm{Hib})$ disease in adults with secondary immunodeficiency in the postHib vaccine era. Clin Vaccine Immunol. 2012;19(5):766-771.

Disclosure of Interests: None declared

DOI: 10.1136/annrheumdis-2020-eular.2635

\section{SAT0113 $\quad$ DISCORDANCE OF CLINICAL REMISSION AND IMAGING REMISSION BY ULTRASONOGRAPHY IN PATIENTS WITH RHEUMATOID ARTHRITIS WITH BIOLOGIC AGENTS}

$\underline{\text { Y. Kondo }}^{1}$, Y. Kaneko ${ }^{1}$, S. Saito ${ }^{1}$, Y. Ohta ${ }^{1}$, K. Sakata ${ }^{1}$, Y. Inoue ${ }^{1}$, C. Takahashi ${ }^{1}$, K. Hiramoto ${ }^{1}$, J. Inamo ${ }^{1}$, T. Takeuchi ${ }^{1}{ }^{1}$ Keio University School of Medicine, Division of Rheumatology, Tokyo, Japan

Background: Residual synovitis can be detected by sensitive modalities such as ultrasonography in patients with rheumatoid arthritis in clinical remission. On the other hand, a previous study has shown that ultrasound-guided treatment provides modest benefit compared to a conventional strategy aiming clinical remission in early patients. It is still unclear how discordant clinical remission is from imaging remission by ultrasonography in patients treated with biologic agents

Objectives: To clarify the discordance between clinical remission and imaging remission in patients with rheumatoid arthritis treated with biologic agents.

Methods: Patients with rheumatoid arthritis who were treated with biologic agents and in clinical remission defined as disease activity score for 28 joints (DAS28)<2.6 were enrolled. All patients were performed comprehensive ultrasound examination of 44 joints as well as physical examinations. Ultrasound images of gray scale (GS) and power doppler (PD) were evaluated with a semi-quantitative score of 0-3. Imaging remission with ultrasound was defined as no PD signal detected in any joints. Clinical information was collected from their medical charts.

Results: A total of 41 patients were enrolled with 22 patients treated with tumor necrosis factor (TNF)-a inhibitors and 19 with interleukin (IL)-6 inhibitors. The mean age, female ratio, the mean disease duration, and the mean duration of clinical remission were 60 years old, $87 \%, 5.1$ years and 11.5 years. The imaging remission by ultrasonography was observed only in $51.2 \%$. When patients were divided according to biologic agents, baseline characteristics including median age, disease duration and clinically remission duration were comparable between both groups, while the rates of seropositivity and the stage of radiological progression was higher in IL-6 group (seropositivity, $p=0.04$; radiological progression, $p=0.02$ ). The mean DAS28 was 1.93 in the TNFa group and 1.02 in the IL- 6 group. The discordance of clinical remission and imaging remission was observed in $28.6 \%$ of the TNFa group and $71.4 \%$ of the IL-6 group $(p=0.03)$. The residual synovitis scores of $G S$ and PD in 44 joints were significantly lower in the TNFa than the IL- 6 group (GS, $1.1 \pm 1.8$ vs $4.7 \pm 4.6, p<0.01 ; \mathrm{PD}, 0.6 \pm 1.3$ vs $3.3 \pm 3.5, \mathrm{p}<0.01$, respectively). A receiver operating characteristic curve demonstrated an optimal score of DAS28 that discriminated imaging remission as 1.89 in the TNFa group and 1.25 in the IL-6 group.

Conclusion: Our results showed that there was substantial discordance between clinical remission and imaging remission, especially in the patients treated with IL-6 inhibitors. In patients treated with biologic agents, clinical remission should be assessed more stringently than the usual 2.6, and ulltrasound-guided management may be useful.

References:

[1] Smolen JS, et al. Ann Rheum Dis 2020;0:1-15.

[2] Iwamoto T, et al. Arthritis Care Res (Hoboken). 2014;66(10):1576-81

[3] Tanaka Y. Ann Rheum Dis 2010;69:1286 -91

[4] Kaneko Y, et al. Ann Rheum Dis 2018;77:1268-1275

[5] Brown AK, et al. Arthritis Rheum 2008;58: 2958 - 67.

Acknowledgments: We would like to thank Harumi Kondo for their assistance.

Disclosure of Interests: Yasushi Kondo: None declared, Yuko Kaneko Speakers bureau: Dr. Kaneko reports personal fees from AbbVie, personal fees from Astellas, personal fees from Ayumi, personal fees from Bristol-Myers Squibb, personal fees from Chugai, personal fees from Eisai, persona fees from Eli Lilly, personal fees from Hisamitsu, personal fees from Jansen, personal fees from Kissei, personal fees from Pfizer, personal fees from Sanofi, personal fees from Takeda, personal fees from Tanabe-Mitsubishi, personal fees from UCB, Shuntaro Saito: None declared, Yuichiro Ohta: None declared, Komei Sakata: None declared, Yumiko Inoue: None declared, Chihiro Takahashi: None declared, Kazuoto Hiramoto: None declared, Jun Inamo: None declared, Tsutomu Takeuchi Grant/research support from: Eisai Co., Ltd, Astellas Pharma Inc., AbbVie GK, Asahi Kasei Pharma Corporation, Nippon Kayaku Co., Ltd, Takeda Pharmaceutical Company Ltd, UCB Pharma, Shionogi \& Co., Ltd., Mitsubishi-Tanabe Pharma Corp., Daiich Sankyo Co., Ltd., Chugai Pharmaceutical Co. Ltd., Consultant of: Chugai Pharmaceutical Co Ltd, Astellas Pharma Inc., Eli Lilly Japan KK, Speakers bureau: AbbVie GK, Eisai Co., Ltd, Mitsubishi-Tanabe Pharma Corporation, Chugai Pharmaceutical Co Ltd, Bristol-Myers Squibb Company, AYUMI Pharmaceutical Corp., Eisai Co., Ltd, Daiichi Sankyo Co., Ltd., Gilead Sciences, Inc., Novartis Pharma K.K., Pfizer Japan Inc., Sanofi K.K., Dainippon Sumitomo Co., Ltd.

DOI: 10.1136/annrheumdis-2020-eular.5048

\section{SAT0114 IMPACT OF DOSE TAPERING REIMBURSEMENT POLICY ON PRESCRIPTION PATTERN OF ADVANCED THERAPY FOR RHEUMATOID ARTHRITIS IN TAIWAN}

C. H. Tang ${ }^{1}$, C. L. Chang ${ }^{1}$, W. Y. Shau ${ }^{2}$, C. Y. Hsin ${ }^{2}$, K. J. LII ${ }^{3}{ }^{1}$ Taipei Medical University, School of Health Care Administration, Taipei City, Taiwan, Republic of China; ${ }^{2}$ Pfizer Ltd., New Taipei City, Taiwan, Republic of China; ${ }^{3}$ National Taiwan University Hospital, Department of Internal Medicine, Taipei City, Taiwan, Republic of China

Background: Rheumatoid arthritis (RA) patients treated with advanced therapy (biologic disease-modifying antirheumatic drugs and targeted synthetic disease-modifying antirheumatic drugs) may be considered dose tapering after reaching treatment goal. ${ }^{1}$ In EULAR 2016 recommendations, dose reduction can be considered if patients reach sustained remission. ${ }^{2} \mathrm{~A}$ dose tapering policy of advanced therapy was introduced in the treatment guideline of RA since 2014 under the National Health Insurance (NHI) in Taiwan. The new reimbursement policy requests the dosage to be tapered in patients who received advanced therapy for 2 years and reached low disease activity defined by DAS28 (ESR).

Objectives: This retrospective study aims to investigate the impact of dose tapering policy on prescription pattern of advanced therapy for RA patients in Taiwan.

Methods: This study was an observational retrospective analysis on the population-based National Health Insurance Research Database (NHIRD) in Taiwan. Patients with RA aged $\geq 18$, initiated an index advanced therapy - abatacept, adalimumab, etanercept, golimumab, tocilizumab, or tofacitinib, during 2011-2017 were included (Figure 1). Patients were followed-up until the index advanced therapy was switched/discontinued or the end of data, whichever came first. The 4-week moving average of proportion of days covered (PDC) of the index therapy within each 12-week period were assessed. The outcome variable was whether dose tapering occurred which was defined as PDC being less than 0.5. The odds ratios (ORs) and the 95\% confidence intervals (Cls) were estimated using Generalized Estimating Equation (GEE) with logistic specification to examine the independent effect of tapering policy and treatment 